\title{
Eldecalcitol prevents endothelial dysfunction in postmenopausal osteoporosis model rats
}

\author{
Kenichi Serizawa', Kenji Yogo', Yoshihito Tashiro², Satoshi Takeda', \\ Ryohei Kawasaki ${ }^{2}$, Ken Aizawa ${ }^{2}$ and Koichi Endo ${ }^{3}$ \\ ${ }^{1}$ Product Research Department, Chugai Pharmaceutical Co., Ltd, 1-135 Komakado, Gotemba, \\ Shizuoka 412-8513, Japan \\ ${ }^{2}$ Product Research Department, Chugai Pharmaceutical Co., Ltd, 200 Kajiwara, Kamakura, \\ Kanagawa 247-8530, Japan \\ ${ }^{3}$ Medical Science Department, Chugai Pharmaceutical Co., Ltd, 2-1-1 Nihonbashi-Muromachi, Chuo-ku, \\ Tokyo 103-8324, Japan
}

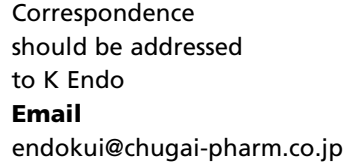

\begin{abstract}
Postmenopausal women have high incidence of cardiovascular events as estrogen deficiency can cause endothelial dysfunction. Vitamin $D$ is reported to be beneficial on endothelial function, but it remains controversial whether vitamin $D$ is effective for endothelial dysfunction under the treatment for osteoporosis in postmenopausal women. The aim of this study was to evaluate the endothelial protective effect of eldecalcitol (ELD) in ovariectomized (OVX) rats. ELD $(20 \mathrm{ng} / \mathrm{kg})$ was orally administrated five times a week for 4 weeks from 1 day after surgery. After that, flow-mediated dilation (FMD) as an indicator of endothelial function was measured by high-resolution ultrasound in the femoral artery of living rats. ELD ameliorated the reduction of FMD in OVX rats. ELD inhibited the increase in NOX4, nitrotyrosine, and $\mathrm{p} 65$ and the decrease in dimer/monomer ratio of nitric oxide synthase in OVX rat femoral arteries. ELD also prevented the decrease in peroxisome proliferator-activated receptor gamma (PPAR $\gamma$ ) in femoral arteries and cultured endothelial cells. Although PPAR $\gamma$ is known to inhibit osteoblastogenesis, ELD understandably increased bone mineral density of OVX rats without increase in PPAR $\gamma$ in bone marrow. These results suggest that ELD prevented the deterioration of endothelial function under condition of preventing bone loss in OVX rats. This endothelial protective effect of ELD might be exerted through improvement of endothelial nitric oxide synthase uncoupling, which is mediated by an antioxidative effect through normalization of vascular PPAR $\gamma / \mathrm{NF}-\mathrm{KB}$ signaling.
\end{abstract}

\author{
Key Words \\ - vitamin D \\ - flow-mediated dilation \\ - endothelial function \\ - estrogen deficiency
}

\section{Introduction}

In postmenopausal women, the risk of cardiovascular event and osteoporosis increases due to diminishing circulating levels of estrogen. Endothelial dysfunction is a powerful surrogate marker of cardiovascular events (Widlansky et al. 2003), and flow-mediated dilation (FMD), which is a useful indicator of endothelial function in clinical settings, is identified as an independent predictor of future cardiovascular events (Yeboah et al. 2007). FMD was identified as the arterial diameter changes after transient ischemia. Transient ischemia leads to reactive hyperemia with increase in flow velocity and vascular wall shear stress. Increased shear stress can induce nitric oxide (NO)

Published by Bioscientifica Ltd. 
production from endothelium, leading to vasodilation (i.e. FMD). In estrogen-deficient postmenopausal women, brachial artery FMD was decreased as compared with premenopausal women (Moreau et al. 2012).

Many reports demonstrated that vitamin D, which is widely used as a supplement for osteoporosis, can exert a beneficial effect on endothelial function. Vitamin D deficiency is related to endothelial dysfunction in various diseases (Yiu et al. 2011, Chitalia et al. 2012) and is an independent predictor of cardiovascular disease and allcause mortality (Dobnig et al. 2008). Vitamin D is effective in improving endothelial function as described above, whereas there are few trials using vitamin $\mathrm{D}$ in postmenopausal women. Although one randomized controlled trial among postmenopausal women with low serum vitamin D status reported no significant improvement of endothelial function by vitamin $\mathrm{D}_{3}$ (Gepner $e$ t al . 2012), further studies are desired to elaborate the effectiveness of vitamin $\mathrm{D}$ on endothelial function in women after menopause (Liu et al. 2013).

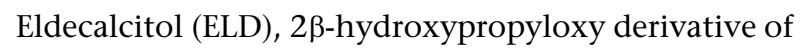
$1 \alpha, 25$-dihydroxyvitamin $\mathrm{D}_{3}$, is currently approved as a drug for treatment of osteoporosis in Japan. Compared with $1 \alpha, 25$-dihydroxyvitamin $\mathrm{D}_{3}$, ELD has a higher affinity for serum vitamin D-binding protein, binds more weakly to vitamin D receptor (VDR), and shows lower potency in suppression of serum PTH (Takahashi 2013). ELD is more efficacious than alfacalcidol ( $1 \alpha$-hydroxyvitamin $\mathrm{D}_{3}$ ) in preventing vertebral and wrist fractures in osteoporotic patients (Matsumoto et al. 2011), however the effect of ELD on endothelial function has not been examined.

In the present study, we examined the effect of ELD on endothelial function by measurement of FMD under condition of preventing bone loss in ovariectomized (OVX) rats.

\section{Materials and methods}

\section{Reagents}

ELD was synthesized in Chugai Pharma Manufacturing Co., Ltd (Tokyo, Japan). ELD was dissolved in mediumchain triglyceride (MCT; Nisshin Oillio, Tokyo, Japan) and diluted to the given concentration.

\section{Animals}

Female Sprague-Dawley rats (Charles River Japan, Yokohama, Japan; 12 weeks old, 200-270 g) were used. All rats were housed in polycarbonate cages with bedding and were fed ordinary laboratory chow and allowed free access to water under a constant $12 \mathrm{~h}$ light:12 h darkness cycle. Rats were randomized into three groups: sham-operated rats receiving vehicle (sham), bilaterally OVX rats receiving vehicle (OVX) and OVX rats receiving ELD (OVX + ELD). ELD (20 ng/kg) or vehicle (MCT) was orally administrated via gavage five times a week for 4 weeks from 1 day after surgery, and then rats were measured FMD (Fig. 1A). This dosage is comparable to clinical dose $(0.75 \mu \mathrm{g}$, daily; Sanford \& McCormack 2011), and prior reports indicated that ELD (15 and $30 \mathrm{ng} / \mathrm{kg}$, daily) increased bone mineral density (BMD) in OVX rats (Sakai et al. 2012). All animal procedures were conducted in accordance with Chugai Pharmaceutical's Ethical Guidelines for Animal Care, and all experimental protocols were approved by the Animal Care Committee of the institution and conformed to the Guide for the Care and Use of Laboratory Animals published by the US National Institutes of Health.

\section{Experimental surgical procedure}

Rats were anesthetized with isoflurane. The lower part of the back was shaved, and a single $0.5-1 \mathrm{~cm}$ incision was made in the skin to expose the back muscles. A small incision was made in the muscles overlying the ovaries on both sides, and the ovaries were isolated, tied with sterile suture, and removed. A sham operation was performed by exposing the ovaries without isolation. An analgesic agent such as flunixin meglumine $(2.5 \mathrm{mg} / \mathrm{kg}$; DS Pharma Animal Health, Osaka, Japan) was subcutaneously administered on the day and 1 day after surgery.

\section{Measurement of body and plasma parameters}

Systolic blood pressure and heart rate were measured by tail-cuff method (BP-98A Softron, Tokyo, Japan) before FMD measurement. Blood samples were collected from abdominal aorta under anesthesia after measurement of FMD, and plasma was stored in a $-80^{\circ} \mathrm{C}$ freezer. Plasma concentrations of calcium and inorganic phosphorus were measured using an autoanalyzer (Hitachi 7170S, Hitachi).

\section{Measurement of FMD}

FMD was measured randomly in rats as described previously (Serizawa et al. 2011, 2012). A graphic representing figure for measurement of FMD is shown in Fig. 1B. Rats were anaesthetized with thiobutabarbital $(120 \mathrm{mg} / \mathrm{kg}$, i.p.; Wako Pure Chemical Industries, Osaka, Japan) with

Published by Bioscientifica Ltd. 
A
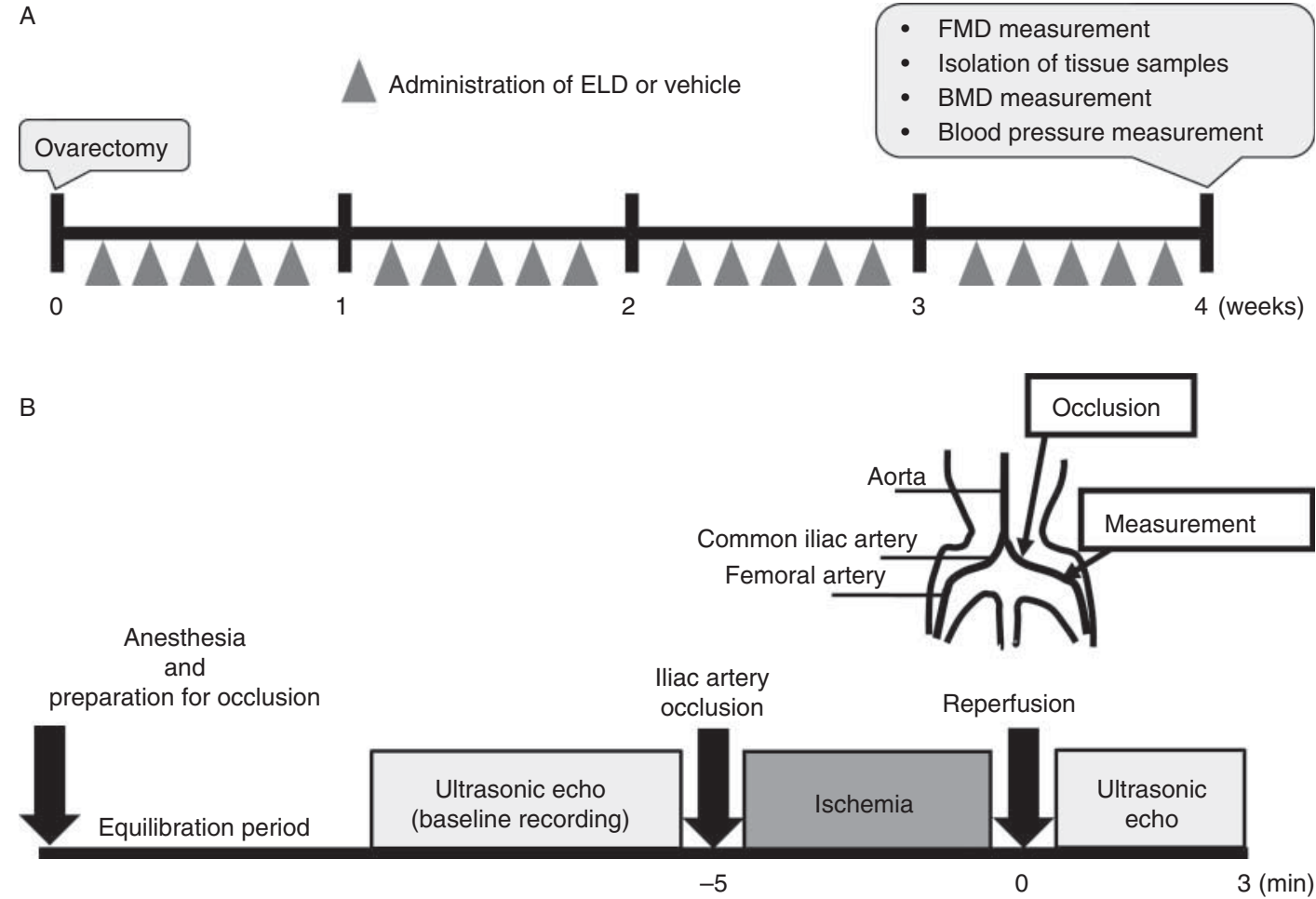

\section{Figure 1}

Diagram of the experimental design. Study designs for treatment of ELD (A) and measurement of FMD (B) were shown as a graphic representing figure. ELD $(20 \mathrm{ng} / \mathrm{kg})$ or vehicle (MCT) was orally administrated via gavage five times a week for 4 weeks from 1 day after surgery, and then rats were

constant monitoring of rectal temperature and monitoring of adequate anesthetic depth by pinching the toe. The animals were kept stable with a heated sheet and warming lamps directed at each rat. Femoral arterial diameter and Doppler flow were measured using a high-resolution ultrasound system (Vevo 770, VisualSonics, Toronto, ON, Canada). The femoral artery was visualized with a $40-\mathrm{MHz}$ transducer. Ischemia and reperfusion of the hindlimb were achieved with a snare occluder positioned around the common iliac artery, through a trans-abdominal access. After an equilibration period, baseline recordings were taken and the common iliac artery was then occluded with the snare occluder. After 5 min of ischemia, the hindlimb was reperfused by releasing the occluder. The changes in flow velocity and the diameter of the femoral artery were monitored at $0,0.5,1,2$, and 3 min after reperfusion. In this study, FMD was decided as the peak changes of femoral artery diameter measured at $0.5 \mathrm{~min}$ after reperfusion, since clinical guideline for FMD assessment defined the peak changes in brachial arterial diameter as an endothelial function, which is occurred about 0.5-1 min after reperfusion (Corretti et al. 2002). measured FMD (A). After baseline recordings, the common iliac artery was occluded for $5 \mathrm{~min}$. After that, the hindlimb was reperfused and the changes in flow velocity and the diameter of the femoral artery were monitored for $3 \mathrm{~min}$ (B).

\section{Western blot analysis}

Rats were euthanized by exsanguination under anesthesia with thiobutabarbital, and femoral arteries and bone marrow were harvested and frozen in liquid $\mathrm{N}_{2}$ immediately after isolation and stored in a $-80^{\circ} \mathrm{C}$ freezer. Equal amounts of protein extracts were separated on SDS-PAGE and immobilized on PVDF membranes. Immunoblotting was performed with anti-peroxisome proliferator-activated receptor gamma (PPAR $\gamma$ ) antibodies (Abcam, Cambridge, UK), anti-endothelial NO synthase (anti-eNOS) antibodies (Santa Cruz Biotechnology), anti-p65 antibodies (Santa Cruz Biotechnology), antiNOX4 antibodies (Epitomics, Burlingame, CA, USA), anti-nitrotyrosine antibodies (Santa Cruz Biotechnology), anti-adipocyte fatty acid-binding protein (A-FABP; Santa Cruz Biotechnology), or anti- $\beta$-actin antibodies (Sigma-Aldrich). To investigate eNOS uncoupling state, low-temperature SDS-PAGE was performed (Grijalva et al. 2008). Briefly, protein extracts were mixed with sample buffer (without $\beta$-mercaptoethanol), and non-boiled samples were separated on SDS-PAGE on ice.

Published by Bioscientifica Ltd 


\section{Measurement of BMD}

After measurement of FMD, rats were euthanized by exsanguination and lumbar vertebrae (L2-L4), and femurs were excised and fixed in $70 \%(\mathrm{v} / \mathrm{v})$ ethanol. The average BMD $\left(\mathrm{mg} / \mathrm{cm}^{2}\right)$ of the lumbar vertebrae (L2-L4) and right femur were measured by dual-energy X-ray absorptiometry (DCS-600EX, Aloka, Tokyo, Japan). During data analysis, the femur was divided into ten equal segments (one to ten) along its major axis. The mean values of the BMD for the three most proximal scanned areas (one to three), those for the next four scanned areas (four to seven), and those for the three most distal areas (eight to ten) were calculated as the densities of the proximal, middle, and distal parts of the femur respectively.

\section{Cell culture and treatment}

Normal human coronary artery endothelial cells (HCAECs) were purchased from Lonza (Walkersville, MD, USA). HCAECs were cultured in endothelial cell basal medium-2 supplemented with $2 \%(\mathrm{v} / \mathrm{v})$ fetal bovine serum (FBS) or endothelial cell basal medium without phenol red indicator (Lonza) supplemented with $2 \%$ (v/v) charcoal stripped FBS (Biological Industries, Beit Haemek, Israel). Medium lacking phenol red was used to remove estrogenic activity because phenol red possesses mild estrogenic activity (Berthois et al. 1986). Charcoal stripped FBS was employed to remove steroid hormones, including estrogen (Horwitz \& McGuire 1978). HCAECs were exposed to ELD $\left(1 \times 10^{-8} \mathrm{M}\right)$, 9-cis retinoic acid (9-cis RA, $1 \times 10^{-6} \mathrm{M}$; Enzo Life Sciences, Farmingdale, NY, USA) or vehicle $(0.1 \%$ (v/v) ethanol). After 7 days, HCAECs were harvested and immediately frozen in liquid $\mathrm{N}_{2}$ and stored in a $-80^{\circ} \mathrm{C}$ freezer until measurement of mRNA by real-time PCR analysis.

\section{Real-time PCR analysis}

Total RNA of HCAECs was isolated using an RNeasy Mini Kit (Qiagen). TaqMan real-time PCR was performed using TaqMan Gene Expression Assays for Ppary (Hs01115513_m1; Applied Biosystems) in an ABI PRISM 7500 Sequence Detection System (Applied Biosystems). Gene expression was normalized to $\beta$-actin (4352935E, Applied Biosystems).

\section{Statistical analyses}

All data are expressed as mean \pm s.E.M. The $n$ values refer to the number of individual animals on which experiments were performed. The statistical significance of differences between each group was determined using Tukey's multiple comparison test, Dunnett's multiple comparison test, and unpaired $t$-test. Test of no correlation was also performed. $P<0.05$ was considered significant. Statistical analyses were performed using SAS Version 8.2 Software or JMP Version 10.0.0 Software (SAS Institute, Cary, NC, USA).

\section{Results}

\section{Physiological parameters}

At the measurement of FMD, the body weight in OVX rats was significantly higher than that in sham-operated rats. There was no significant influence of treatment with ELD on body weight. Systolic blood pressure and heart rate were not changed among the groups. Ovariectomy did not alter plasma calcium and phosphorus levels. Treatment with ELD increased plasma calcium level within normal limit but not phosphorus levels in OVX rats (Table 1).

\section{FMD in femoral arteries of OVX rats}

Reperfusion after $5 \mathrm{~min}$ ischemia instantaneously increased flow velocity (i.e. reactive hyperemia) compared with baseline femoral artery flow. This reactive hyperemia was peaked at just after reperfusion and rapidly decayed to baseline values at around $3 \mathrm{~min}$. Reactive hyperemia did not differ among the groups (Fig. 2A, B and C). The increase in flow velocity was associated with a delayed increase in femoral arterial vasodilation that peaked at 0.5 min after reperfusion (Fig. 2D and E). This delayed vasodilation was observed as FMD. FMD was significantly decreased in OVX rats compared with sham-operated

Table 1 Physiological parameters in sham-operated, OVX, or OVX rats treated with ELD for 4 weeks.

\begin{tabular}{|c|c|c|c|}
\hline & Sham & ovx & OVX + ELD \\
\hline$n$ & 7 & 10 & 8 \\
\hline Body weight (g) & $294.0 \pm 5.5$ & $340.2 \pm 4.7 *$ & $350.4 \pm 5.8^{*}$ \\
\hline $\begin{array}{l}\text { Systolic blood pressure } \\
(\mathrm{mmHg})\end{array}$ & $129.3 \pm 4.2$ & $118.5 \pm 2.2$ & $121.4 \pm 4.4$ \\
\hline Heart rate (b.p.m.) & $398.3 \pm 8.3$ & $367.4 \pm 8.5$ & $405.2 \pm 24.8$ \\
\hline Plasma calcium (mg/dl) & $8.8 \pm 0.1$ & $8.5 \pm 0.1$ & $9.1 \pm 0.2^{\dagger}$ \\
\hline $\begin{array}{l}\text { Plasma phosphorus } \\
\text { (mg/dl) }\end{array}$ & $6.5 \pm 0.3$ & $6.4 \pm 0.2$ & $6.7 \pm 0.2$ \\
\hline
\end{tabular}

${ }^{*} P<0.05$ vs sham and ${ }^{\dagger} P<0.05$ vs $\mathrm{OVX}$ by Tukey's multiple comparison test.

Published by Bioscientifica Ltd. 

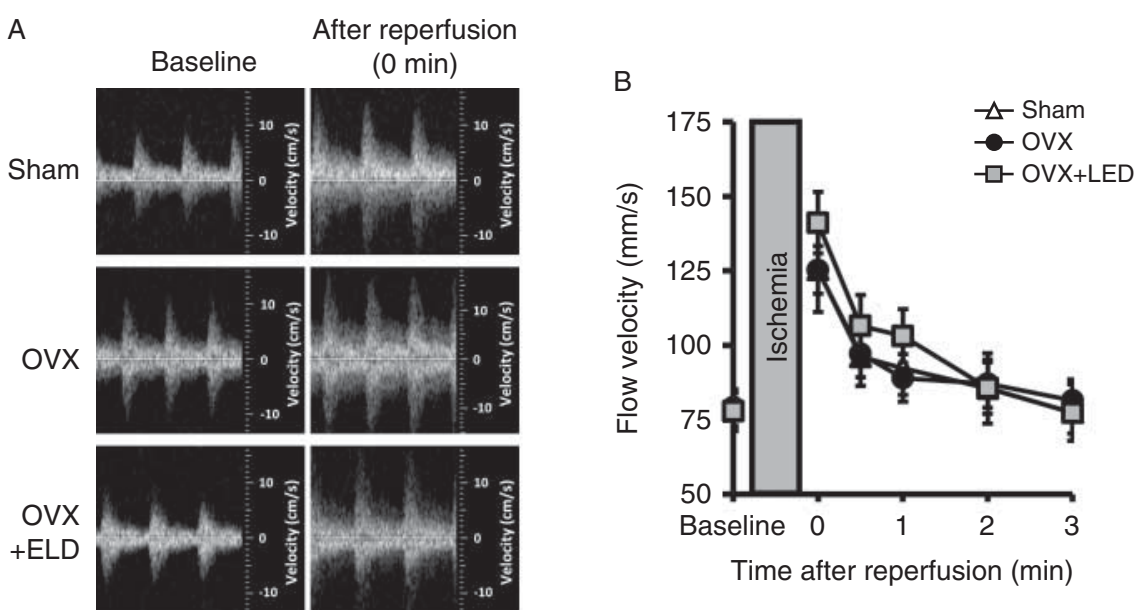

Time after reperfusion $(\mathrm{min})$

After reperfusion
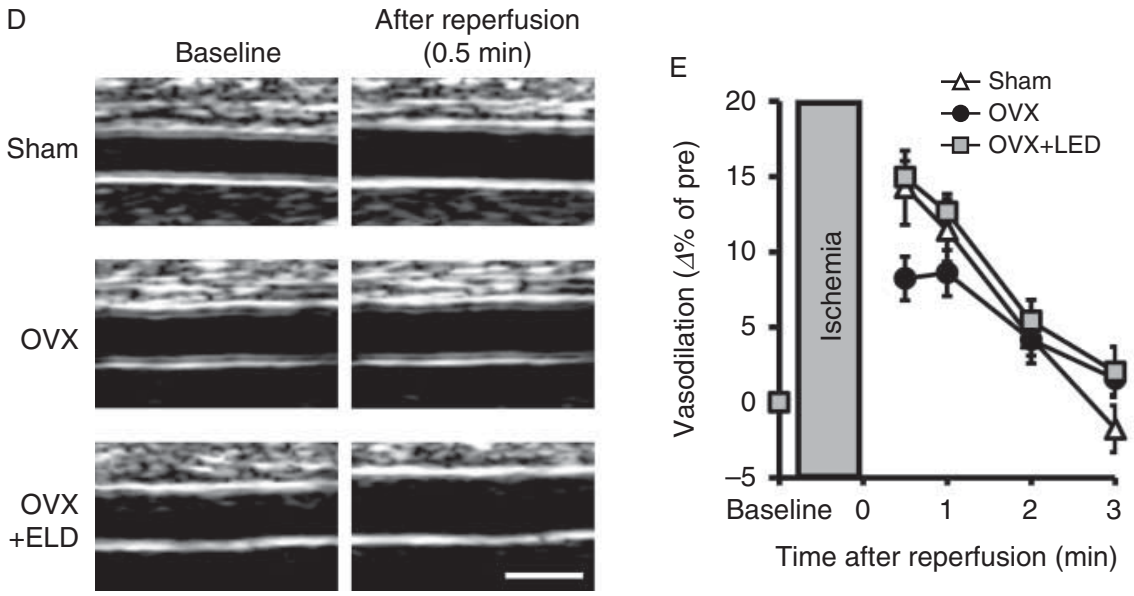
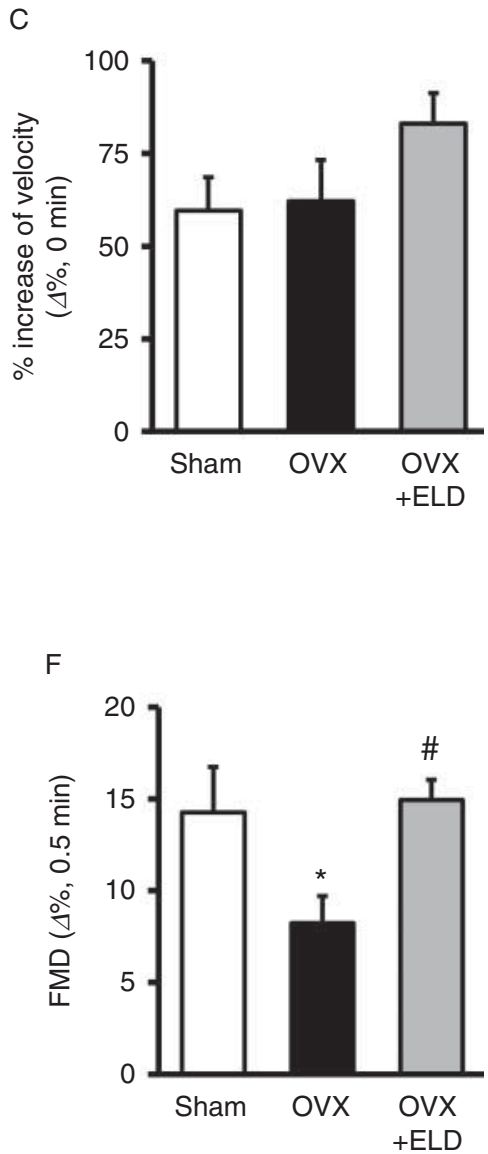

Figure 2

Post-ischemic reactive hyperemia and flow-mediated dilation in femoral arteries of OVX rats. Ultrasound measurements were performed at femoral arteries. Representative ultrasound images show Doppler flow velocity (A) and femoral artery (D). Scale bar $=0.5 \mathrm{~mm}$. Flow velocity was peaked at just after reperfusion (B), and increase rate of flow velocity was evaluated at

rats despite the same level of reactive hyperemia, i.e., same stimulation to endothelium. ELD prevented the reduction of FMD in OVX rats to almost same level as in sham-operated rats (sham, 14.3 \pm 2.5 ; OVX, 8.2 \pm 1.5 ;

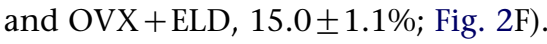

\section{Oxidative stress in femoral arteries of OVX rats}

Oxidative stress could play a crucial role in endothelial dysfunction. In OVX rats, the expression of NOX4, which is NADPH oxidase component, was significantly increased in femoral arteries, and this increase was consistent with augmented oxidative stress as reflected by the nitrotyrosine accumulation in femoral arteries. ELD prevented
$0 \mathrm{~min}(\mathrm{C})$. Vasodilation was peaked at $0.5 \mathrm{~min}$ after reperfusion (E), and peak vasodilation was evaluated as FMD (F). ELD prevented the reduction of FMD in OVX rats. ${ }^{*} P<0.05$ vs sham and ${ }^{\#} P<0.05$ vs OVX by Tukey's multiple comparison test $(n=7-10)$.

the increase in the expression of NOX4 and nitrotyrosine accumulation in OVX rat femoral arteries (Fig. 3A and B). Since NOX4 expression can be regulated by NF- $\mathrm{B}$, we also detected p65 expression which is NF- $\kappa \mathrm{B}$ subunit. The expression of p65 was significantly increased in femoral arteries of OVX rats compared with sham-operated rats. ELD significantly decreased p65 expression (Fig. 3C).

\section{Expression of PPAR $\gamma$ in femoral arteries of OVX rats or HCAECs}

PPAR $\gamma$ is a key molecule in regulating NOX4 expression through NF- $\kappa$ B. In femoral arteries of OVX rats, ELD restored the decrease in PPAR $\gamma$ expression (Fig. 4A).

Published by Bioscientifica Ltd 
A
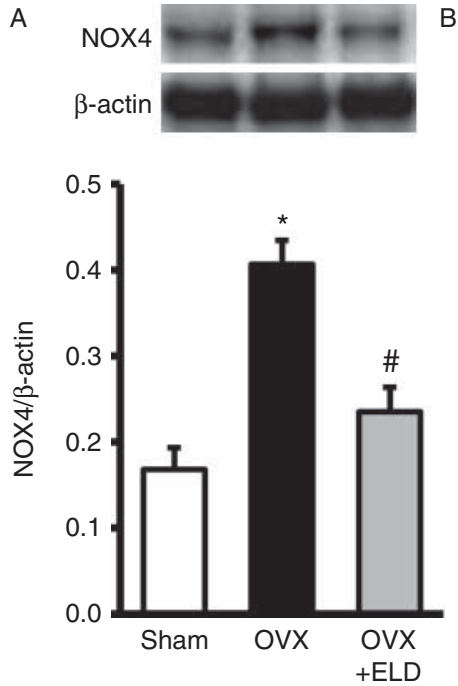

B
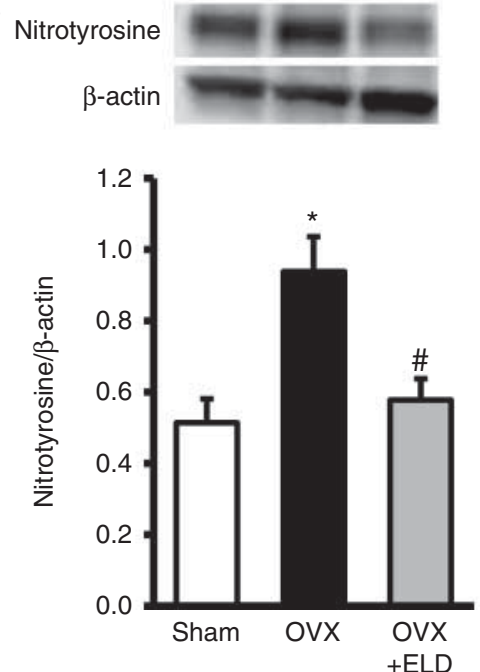

C
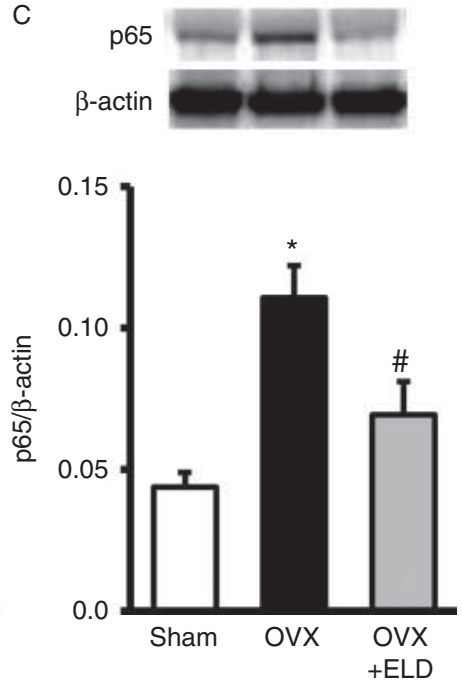

Figure 3

Oxidative stress in femoral arteries of OVX rats. The expression of NOX4 was increased in OVX rat femoral arteries, and ELD prevented it (A).

Nitrotyrosine accumulation was also increased in OVX rat femoral arteries,

In HCAECs, Ppary mRNA was significantly decreased by culture in phenol red free medium, and ELD prevented it (Fig. 4B). VDR is known to heterodimerize with retinoid $X$ receptor (RXR), and RXR ligand can activate PPAR $\gamma$ (Sanz et al. 2012). Ppary mRNA, however, was not changed in HCAECs by 9-cis RA, which is a ligand of RXR $\alpha$.

\section{eNOS uncoupling in femoral arteries of OVX rats}

Oxidative stress leads to eNOS uncoupling state. In this study, eNOS dimer/monmer ratio was significantly decreased in OVX rat femoral arteries. This result means the increase in eNOS uncoupling. ELD significantly restored the decrease in eNOS dimer/monomer ratio in OVX rat femoral arteries (Fig. 5).

\section{BMD in the lumbar spine and the femur}

To confirm the effect of ELD on bone loss, BMD of L2-L4 lumbar vertebrae and the femur were measured. In rats treated with ELD for 4 weeks, BMD of lumbar vertebrae and distal segment of femur were significantly increased compared with OVX rats (Fig. 6A and B).

\section{Expression of PPAR $\gamma$ in bone marrow of OVX rats}

Activation of arterial PPAR $\gamma$ exerts vasoprotective effects, whereas activation of PPAR $\gamma$ in bone marrow is and ELD prevented it (B). ELD also inhibited the increase in 065 expression in femoral arteries of OVX rats (C). ${ }^{*} P<0.05$ vs sham and ${ }^{\#} P<0.05$ vs OVX by Tukey's multiple comparison test $(n=7-10)$.

known to be a risk factor of bone loss and fracture via increased myeloid adipogenesis at the expense of osteoblast formation. Contrary to the effect of ELD in femoral arteries, ELD showed a tendency to prevent the increase in PPAR $\gamma$ expression in bone marrow (Fig. 7A). The expression of PPAR $\gamma$ in bone marrow showed positive correlation with the expression of A-FABP that is expressed in adipocytes (Fig. 7B).

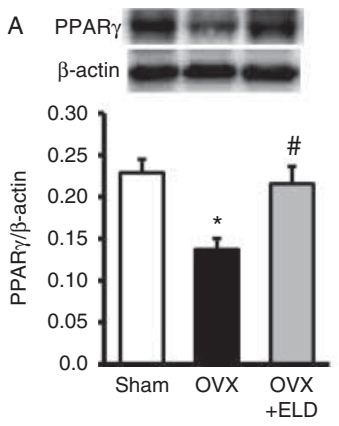

B

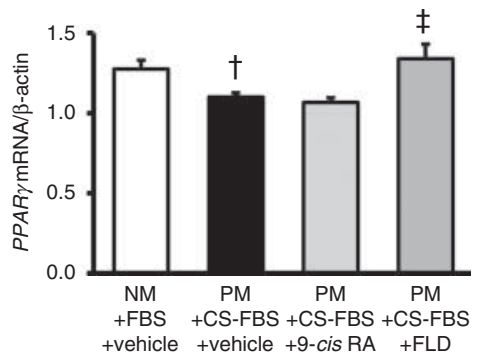

Figure 4

Expression of PPAR $\gamma$ in femoral arteries of OVX rats and HCAECs. The expression of PPAR $\gamma$ was decreased in OVX rat femoral arteries, and ELD prevented it (A). PPAR $\gamma$ mRNA was also reduced in HCAECs cultured in phenol red free medium (PM) + charcoal stripped FBS (CS-FBS) compared with HCAECs cultured in normal medium (NM) + FBS (B). ELD inhibited the decrease in mRNA of PPAR $\gamma$ whereas 9-cis RA did not. ${ }^{*} P<0.05$ vs sham and ${ }^{\#} P<0.05$ vs OVX by Tukey's multiple comparison test $(n=7-10) .{ }^{\dagger} P<0.05$ vs $\mathrm{NM}+\mathrm{FBS}+$ vehicle by unpaired $t$-test, ${ }^{\ddagger} P<0.05$ vs PM + CS-FBS + vehicle by Dunnett's multiple comparison test $(n=4)$.

Published by Bioscientifica Ltd 


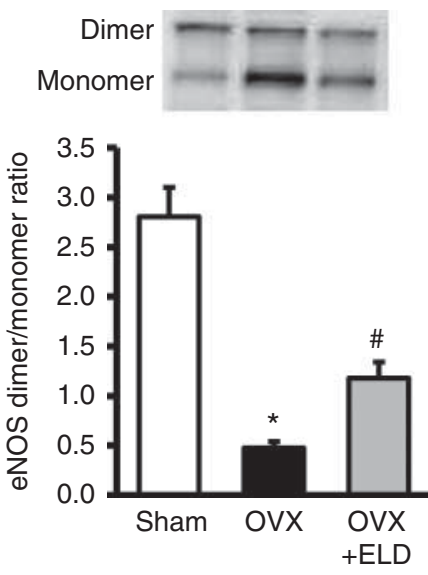

Figure 5

eNOS uncoupling in femoral arteries of OVX rats. Dimer and monomer eNOS were detected by low-temperature SDS-PAGE. In femoral arteries of OVX rats, eNOS uncoupling, indicated as reduction of dimer/monomer ratio, was decreased, and ELD prevented it. ${ }^{*} P<0.05$ vs sham and ${ }^{\#} P<0.05$ vs OVX by Tukey's multiple comparison test $(n=7-10)$.

\section{Discussion}

Clinical dose of ELD prevents reduction of FMD in femoral arteries of OVX rats. ELD inhibited deterioration of oxidative stress with higher PPAR $\gamma$ expression and lower $\mathrm{NF}-\kappa \mathrm{B}$ expression than those in femoral arteries of vehicletreated OVX rats. ELD also ameliorated eNOS uncoupling state in OVX rat femoral arteries. ELD naturally increased BMD of lumbar vertebrae and femur with an increase in plasma calcium level. The mean value of plasma calcium was still within the normal range. In bone marrow of OVX rats, ELD did not increase PPAR $\gamma$ expression, unlike in femoral arteries. Therefore, it is suggested that ELD may prevent endothelial dysfunction through the reduction of oxidative stress via normalization of vascular PPAR $\gamma / \mathrm{NF}$ $\kappa \mathrm{B}$ pathway in OVX rats. These beneficial effects of ELD correspond to previous report that calcitriol restored renovascular function in OVX rats (Dong et al. 2013). In addition, this is the first report indicated that in vivo endothelial function was evaluated by measurement of FMD under the study design, in which ELD could prevent bone loss in OVX rats.

\section{Involvement of oxidative stress in endothelial dysfunction}

A common mechanism underlying endothelial dysfunction is increased oxidative stress derived from vascular NADPH oxidase in vascular tissue. Recent reports demonstrated that increased oxidative stress via NADPH oxidase induced endothelial dysfunction in OVX rat (Camporez et al. 2011). Increased reactive oxygen species (ROS) production via NADPH oxidase can limit NO availability. Superoxide rapidly reacts with NO, resulting in formation of the peroxynitrite anion and loss of the amount of available NO (Gryglewski et al. 1986). Furthermore, increased peroxynitrite can oxidize avidly tetrahydrobiopterin $\left(\mathrm{BH}_{4}\right)$ to dihydrobiopterin. Under conditions of $\mathrm{BH}_{4}$ deficiency, eNOS is in an uncoupled state, resulting in production of superoxide rather than NO (Landmesser et al. 2004). This reduction of NO availability can cause endothelial dysfunction. In clinical settings, enhanced NO availability by estrogen replacement therapy improved endothelial function in postmenopausal women (Tagawa et al. 1997). In the present study, we demonstrated that FMD was decreased in OVX rat femoral arteries, along with the increase in NOX4 expression. NOX4 is a component of NADPH oxidase and is highly expressed in vascular wall cells including smooth muscle and endothelial cells. In contrast to the other NOX homologs, NOX4 is constitutively active (Ambasta et al. 2004), and the increase in NOX4 expression contributes to the activity (Serrander et al. 2007). Correspondingly, oxidative stress assessed by nitrotyrosine accumulation, as a marker of peroxynitrite, was increased in OVX rat femoral arteries. This result was associated with deterioration of eNOS uncoupling assessed by eNOS dimer/monomer ratio. ELD significantly improved those changes in OVX rat femoral arteries. Plasma NOx, which is NO metabolite, and plasma malondialdehyde as a marker of oxidative stress were not changed among the groups (data not shown). Therefore, it is suggested that ELD prevented endothelial dysfunction through the correction of NO availability by inhibition of oxidative stress in femoral arteries of OVX rats. These results are corresponding to the report that
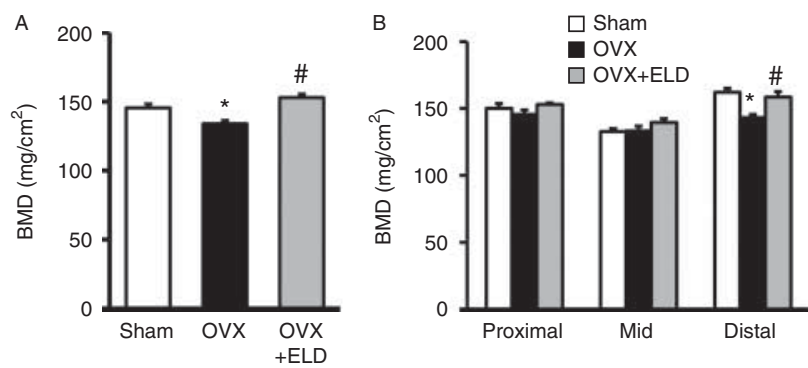

Figure 6

BMD in lumbar vertebrae and femur of OVX rats. BMD was measured in L2-L4 lumbar vertebrae (A) and femur (B). ELD increased BMD in lumbar vertebrae and femur of OVX rats. ${ }^{*} P<0.05$ vs sham and ${ }^{\#} P<0.05$ vs OVX by Tukey's multiple comparison test $(n=7-8)$.

Published by Bioscientifica Ltd. 

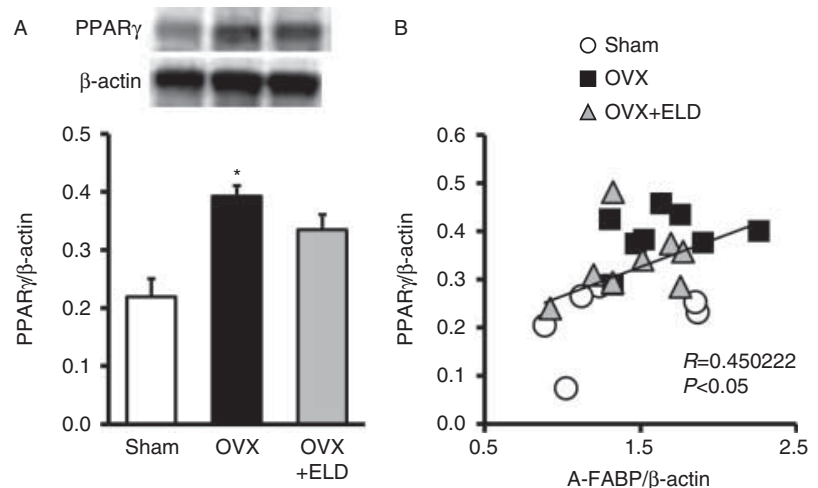

Figure 7

Expression of PPAR $\gamma$ in bone marrow of OVX rats. The expression of PPAR $\gamma$ was increased in bone marrow of OVX rat, and ELD showed a tendency to reduce it. The expression of PPAR $\gamma$ in bone marrow showed positive correlation with the expression of A-FABP. ${ }^{*} P<0.05$ vs sham by Tukey's multiple comparison test $(n=6-8)$.

1 $\alpha, 25$-dihydroxyvitamin $\mathrm{D}_{3}$ protects vascular function through the inhibition of oxidative stress such as NOX4 and nitrotyrosine in hypertensive patients and animals (Dong et al. 2012). We recently reported that the other vitamin D analog, 22-oxacalcitriol, also prevents progression of endothelial dysfunction through antioxidative effects in type 2 diabetic rats (Hirata et al. 2013).

\section{Involvement of PPAR $\gamma$ in endothelial dysfunction of OVX rats}

PPAR $\gamma$ is expressed in vascular endothelial cells and has been recognized to have vasculoprotective effects. Pioglitazone, which is a PPAR $\gamma$ agonist, improved endothelial function in coronary arterial disease patients (Rizza et al. 2011). Beyer et al. (2008) demonstrated that endotheliumspecific interference with PPAR $\gamma$ caused endothelial dysfunction. In OVX mice, PPAR $\gamma$ expression was significantly reduced in aortic tissue, and PPAR $\gamma$ agonist prevented endothelial dysfunction in isolated aortic ring preparations (Tiyerili et al. 2012). Recent studies indicated that PPAR $\gamma$ regulated NOX4 expression through NF- $\kappa$ B (Lu et al. 2010). In this study, we demonstrated that PPAR $\gamma$ expression was decreased in OVX rat femoral arteries accompanied by the increase in p65 and NOX4 expression. ELD restored PPAR $\gamma$ expression and prevented the increase in p65 and NOX4 expression in femoral arteries of OVX rats. The increase in Ppary mRNA by ELD was also observed in HCAECs cultured in phenol red free medium. Therefore, it is suggested that ELD prevented endothelial dysfunction through the improvement of endothelial PPAR $\gamma / \mathrm{NF}-\kappa \mathrm{B} / \mathrm{NOX} 4$ pathway in OVX rats.
PPAR $\gamma$ has also been known to be involved in bone homeostasis. Mice treated with rosiglitazone exhibited bone loss accompanied by an increase in marrow adipocyte formation and a decrease in the ratio of osteoblasts to osteoclasts (Ali et al. 2005). Pharmacological inhibition of PPAR $\gamma$ increased osteoblastogenesis and bone mass (Duque et al. 2013). In this study, the expression of PPAR $\gamma$ was significantly increased in bone marrow of OVX rats associated with bone loss. ELD increased $\mathrm{BMD}$ and showed tendency to reduce the expression of PPAR $\gamma$ in bone marrow of OVX rats. The expression of PPAR $\gamma$ in bone marrow was positively associated with A-FABP expression. Our results are consistent with earlier report that $1 \alpha, 25$-dihydroxyvitamin $\mathrm{D}_{3}$ inhibited bone marrow adipogenesis by decreasing PPAR $\gamma$ expression in senescence accelerated mice (Duque et al. 2004). Therefore, the present study suggests that estrogen deficiency showed regiospecific influence in the PPAR $\gamma$ expression, and ELD exerts bone mass increasing effect and endothelial protective effect by organ-specific regulation of $\operatorname{PPAR} \gamma$.

\section{Limitations of this study}

OVX model is the most commonly used and extensively studied animal model of postmenopausal osteoporosis. FDA guidelines state that OVX rat model is appropriate for evaluation of agents to prevent bone loss in postmenopausal women (Thompson et al. 1995). However, OVX model does not completely replicate postmenopausal women in terms of the pattern of estrogen deficiency. Because it is known that surgical ovariectomy in premenopausal women also caused endothelial dysfunction (Takahashi et al. 2007), it is possible that endothelial dysfunction in OVX rats is more close to that in women who underwent surgical ovariectomy. Additional examination may be needed to clarify whether ELD can prevent the deterioration of endothelial function by a gradual estrogen depletion.

Obesity is known to be a risk factor of cardiovascular disease (CVD) events. On the other hand, it is also reported that plasma adipocytokine levels such as leptin were positively associated with vascular endothelial function in overweight patients (Morioka et al. 2014). Therefore, adipose tissue may have some influence on endothelial function. Tarcin et al. (2009) demonstrated that vitamin D replacement increased serum leptin levels accompanied by improving FMD in asymptomatic subjects. In the present study, ELD did not change the increased body weight in OVX rats. However, it is unclear whether ELD

Published by Bioscientifica Ltd 
affected the function of adipose tissue such as the capacity for adipocytokine production. Although endothelial VDR is important for endothelial function (Ni et al. 2014), it is not yet known which mechanism is more important in endothelial protection in OVX rats, endothelial VDR activation, or adipocytokine production.

There are many reports demonstrated that vitamin $\mathrm{D}_{3}$ is beneficial for endothelial function. On the other hand, it is reported that vitamin $\mathrm{D}_{3}$ has less to do with vascular function. Siasos et al. (2014) indicated that vitamin $\mathrm{D}_{2}$ concentrations were positively associated with endothelial function in coronary artery disease patients, although vitamin D status was not associated with arterial wall properties. Because vitamin $\mathrm{D}_{2}$ also exerts its effect via VDR, VDR activation is certainly important for endothelial function. However, it may be controversial that vitamin $\mathrm{D}_{3}$ is absolutely essential for endothelial function in clinical settings.

\section{Conclusion}

The present study demonstrated that ELD prevented the deterioration of endothelial function in OVX rats through improvement of eNOS uncoupling by an antioxidative effect. This antioxidative effect may be mediated by normalization of vascular PPAR $\gamma / \mathrm{NF}-\kappa \mathrm{B}$ signaling pathway.

\section{Declaration of interest}

All authors are employees of Chugai Pharmaceutical Co., Ltd.

\section{Funding}

This work was supported by Chugai Pharmaceutical Co., Ltd.

\section{Author contribution statement}

Study design: $\mathrm{K} \mathrm{S}, \mathrm{K} Y$, and $\mathrm{K} \mathrm{E}$; study conduct: $\mathrm{KS}, \mathrm{KY}, \mathrm{YT}$, S T, R K, and K A; data collection and analysis: $K S$ and $S T$; data interpretation: $K S, K Y, Y T$, $S T, R K, K A$, and $K E$; drafting manuscript: $K S$; revising manuscript content: $K S, K Y$, and $K E$; approving final version of manuscript: $K E$; and $K E$ takes responsibility for the integrity of the data analysis.

\section{Acknowledgements}

The authors are grateful to Mika Yagoto for her technical assistance.

\section{References}

Ali AA, Weinstein RS, Stewart SA, Parfitt AM, Manolagas SC \& Jilka RL 2005 Rosiglitazone causes bone loss in mice by suppressing osteoblast differentiation and bone formation. Endocrinology 146 1226-1235. (doi:10.1210/en.2004-0735)
Ambasta RK, Kumar P, Griendling KK, Schmidt HH, Busse R \& Brandes RP 2004 Direct interaction of the novel Nox proteins with p22phox is required for the formation of a functionally active NADPH oxidase. Journal of Biological Chemistry 279 45935-45941. (doi:10.1074/jbc. M406486200)

Berthois Y, Katzenellenbogen JA \& Katzenellenbogen BS 1986 Phenol red in tissue culture media is a weak estrogen: implications concerning the study of estrogen-responsive cells in culture. PNAS 83 2496-2500. (doi:10.1073/pnas.83.8.2496)

Beyer AM, de Lange WJ, Halabi CM, Modrick ML, Keen HL, Faraci FM \& Sigmund CD 2008 Endothelium-specific interference with peroxisome proliferator activated receptor $\gamma$ causes cerebral vascular dysfunction in response to a high-fat diet. Circulation Research 103 654-661. (doi:10.1161/CIRCRESAHA.108.176339)

Camporez JP, Akamine EH, Davel AP, Franci CR, Rossoni LV \& Carvalho CR 2011 Dehydroepiandrosterone protects against oxidative stressinduced endothelial dysfunction in ovariectomized rats. Journal of Physiology 589 2585-2596. (doi:10.1113/jphysiol.2011.206078)

Chitalia N, Recio-Mayoral A, Kaski JC \& Banerjee D 2012 Vitamin D deficiency and endothelial dysfunction in non-dialysis chronic kidney disease patients. Atherosclerosis 220 265-268. (doi:10.1016/ j.atherosclerosis.2011.10.023)

Corretti MC, Anderson TJ, Benjamin EJ, Celermajer D, Charbonneau F, Creager MA, Deanfield J, Drexler H, Gerhard-Herman M, Herrington D et al. 2002 Guidelines for the ultrasound assessment of endothelialdependent flow-mediated vasodilation of the brachial artery: a report of the International Brachial Artery Reactivity Task Force. Journal of the American College of Cardiology 39 257-265. (doi:10.1016/S0735-1097 (01)01746-6)

Dobnig H, Pilz S, Scharnagl H, Renner W, Seelhorst U, Wellnitz B, Kinkeldei J, Boehm BO, Weihrauch G \& Maerz W 2008 Independent association of low serum 25-hydroxyvitamin d and 1,25-dihydroxyvitamin D levels with all-cause and cardiovascular mortality. Archives of Internal Medicine 168 1340-1349. (doi:10.1001/archinte.168.12.1340)

Dong J, Wong SL, Lau CW, Lee HK, Ng CF, Zhang L, Yao X, Chen ZY, Vanhoutte PM \& Huang Y 2012 Calcitriol protects renovascular function in hypertension by down-regulating angiotensin II type 1 receptors and reducing oxidative stress. European Heart Journal 33 2980-2990. (doi:10.1093/eurheartj/ehr459)

Dong J, Wong SL, Lau CW, Liu J, Wang YX, He ZD, Ng CF, Chen ZY, Yao X, $\mathrm{Xu}$ A et al. 2013 Carcitriol restores renovascular function in estrogendeficient rats through downregulation of cyclooxygenase- 2 and the thromboxane-prostanoid receptor. Kidney International 84 54-63. (doi:10.1038/ki.2013.12)

Duque G, Macoritto M \& Kremer R 2004 1,25(OH) ${ }_{2} \mathrm{D}_{3}$ inhibits bone marrow adipogenesis in senescence accelerated mice (SAM-P/6) by decreasing the expression of peroxisome proliferator-activated receptor $\gamma 2$ (PPAR $\gamma 2$ ). Experimental Gerontology 39 333-338. (doi:10.1016/ j.exger.2003.11.008)

Duque G, Li W, Vidal C, Bermeo S, Rivas D \& Henderson J 2013 Pharmacological inhibition of PPAR $\gamma$ increases osteoblastogenesis and bone mass in male C57BL/6 mice. Journal of Bone and Mineral Research 28 639-648. (doi:10.1002/jbmr.1782)

Gepner AD, Ramamurthy R, Krueger DC, Korcarz CE, Binkley N \& Stein JH 2012 A prospective randomized controlled trial of the effects of vitamin D supplementation on cardiovascular disease risk. PLOS ONE 7 e36617. (doi:10.1371/journal.pone.0036617)

Grijalva J, Hicks S, Zhao X, Medikayala S, Kaminski PM, Wolin MS \& Edwards JG 2008 Exercise training enhanced myocardial endothelial nitric oxide synthase (eNOS) function in diabetic Goto-Kakizaki (GK) rats. Cardiovascular Diabetology 7 34. (doi:10.1186/1475-2840-7-34)

Gryglewski RJ, Palmer RM \& Moncada S 1986 Superoxide anion is involved in the breakdown of endothelium-derived vascular relaxing factor. Nature 320 454-456. (doi:10.1038/320454a0)

Hirata M, Serizawa K, Aizawa K, Yogo K, Tashiro Y, Takeda S, Moriguchi Y, Endo K \& Fukagawa M 2013 22-Oxacalcitriol prevents progression of 
endothelial dysfunction through antioxidative effects in rats with type 2 diabetes and early-stage nephropathy. Nephrology, Dialysis, Transplantation 28 1166-1174. (doi:10.1093/ndt/gfs536)

Horwitz KB \& McGuire WL 1978 Estrogen control of progesterone receptor in human breast cancer. Correlation with nuclear processing of estrogen receptor. Journal of Biological Chemistry 253 2223-2228.

Landmesser U, Hornig B \& Drexler H 2004 Endothelial function: a critical determinant in atherosclerosis? Circulation 109 II27-II33. (doi:10.1161/ 01.CIR.0000129501.88485.1f)

Liu ZM, Woo J, Wu SH \& Ho SC 2013 The role of vitamin D in blood pressure, endothelial and renal function in postmenopausal women. Nutrients 5 2590-2610. (doi:10.3390/nu5072590)

Lu X, Murphy TC, Nanes MS \& Hart CM $2010 \operatorname{PPAR}\{\gamma\}$ regulates hypoxiainduced Nox4 expression in human pulmonary artery smooth muscle cells through NF-\{ $\kappa\}$ B. American Journal of Physiology. Lung Cellular and Molecular Physiology 299 L559-L566. (doi:10.1152/ajplung.00090.2010)

Matsumoto T, Ito M, Hayashi Y, Hirota T, Tanigawara Y, Sone T, Fukunaga M, Shiraki M \& Nakamura T 2011 A new active vitamin D3 analog, eldecalcitol, prevents the risk of osteoporotic fractures-a randomized, active comparator, double-blind study. Bone 49 605-612. (doi:10.1016/j.bone.2011.07.011)

Moreau KL, Hildreth KL, Meditz AL, Deane KD \& Kohrt WM 2012 Endothelial function is impaired across the stages of the menopause transition in healthy women. Journal of Clinical Endocrinology and Metabolism 97 4692-4700. (doi:10.1210/jc.2012-2244)

Morioka T, Emoto M, Yamazaki Y, Kawano N, Imamura S, Numaguchi R, Urata H, Motoyama K, Mori K, Fukumoto S et al. 2014 Leptin is associated with vascular endothelial function in overweight patients with type 2 diabetes. Cardiovascular Diabetology 13 10. (doi:10.1186/ 1475-2840-13-10)

Ni W, Watts SW, Ng M, Chen S, Glenn DJ \& Gardner DG 2014 Elimination of vitamin D reveptor in vascular endothelial cells alters vascular function. Hypertension 64 1290-1298. (doi:10.1161/ HYPERTENSIONAHA.114.03971)

Rizza S, Cardellini M, Porzio O, Pecchioli C, Savo A, Cardolini I, Senese N, Lauro D, Sbraccia P, Lauro R et al. 2011 Pioglitazone improves endothelial and adipose tissue dysfunction in pre-diabetic CAD subjects. Atherosclerosis 215 180-183. (doi:10.1016/j.atherosclerosis. 2010.12.021)

Sakai S, Endo K, Takeda S, Mihara M \& Shiraishi A 2012 Combination therapy with eldecalcitol and alendronate has therapeutic advantages over monotherapy by improving bone strength. Bone 50 1054-1063. (doi:10.1016/j.bone.2012.02.005)

Sanford M \& McCormack PL 2011 Eldecalcitol: a review of its use in the treatment of osteoporosis. Drugs $\mathbf{7 1}$ 1755-1770. (doi:10.2165/ 11206790-000000000-00000)

Sanz MJ, Albertos F, Otero E, Juez M, Morcillo EJ \& Piqueras L 2012 Retinoid $\mathrm{X}$ receptor agonists impair arterial mononuclear cell recruitment through peroxisome proliferator-activated receptor- $\gamma$ activation. Journal of Immunology 189 411-424. (doi:10.4049/jimmunol.1102942)

Serizawa K, Yogo K, Aizawa K, Tashiro Y \& Ishizuka N 2011 Nicorandil prevents endothelial dysfunction due to antioxidative effects via normalisation of NADPH oxidase and nitric oxide synthase in streptozotocin diabetic rats. Cardiovascular Diabetology 10105. (doi:10.1186/1475-2840-10-105)
Serizawa K, Yogo K, Aizawa K, Tashiro Y, Takahari Y, Sekine K, Suzuki T, Ishizuka N \& Ishida H 2012 Paclitaxel-induced endothelial dysfunction in living rats is prevented by nicorandil via reduction of oxidative stress. Journal of Pharmacological Sciences 119 349-358. (doi:10.1254/ jphs.12067FP)

Serrander L, Cartier L, Bedard K, Banfi B, Lardy B, Plastre O, Sienkiewicz A, Forro L, Schlegel W \& Krause KH 2007 NOX4 activity is determined by mRNA levels and reveals a unique pattern of ROS generation. Biochemical Journal 406 105-114. (doi:10.1042/BJ20061903)

Siasos G, Tousoulis D, Oikonomou E, Maniatis K, Kioufis S, Kokkou E, Vavuranakis M, Zaromitidou M, Kassi E, Miliou A et al. 2014 Vitamin D3, D2 and arterial wall properties in coronary artery disease. Current Pharmaceutical Design 20 5914-5918. (doi:10.2174/ 1381612820666140619122937)

Tagawa H, Shimokawa H, Tagawa T, Kuroiwa-Matsumoto M, Hirooka Y \& Takeshita A 1997 Short-term estrogen augments both nitric oxidemediated and non-nitric oxide-mediated endothelium-dependent forearm vasodilation in postmenopausal women. Journal of Cardiovascular Pharmacology 30 481-488. (doi:10.1097/00005344199710000-00012)

Takahashi N 2013 Mechanism of inhibitory action of eldecalcitol, an active vitamin $\mathrm{D}$ analog, on bone resorption in vivo. Journal of Steroid Biochemistry and Molecular Biology 136 171-174. (doi:10.1016/j.jsbmb. 2012.11.010)

Takahashi K, Mori-Abe A, Takata K, Ohta T, Kawagoe J, Tsutsumi S, Ohmichi M \& Kurachi H 2007 Raloxifene improves the ovariectomyinduced impairment in endothelium-dependent vasodilation. Menopause 14 656-661. (doi:10.1097/01.gme.0000248704.30204.33)

Tarcin O, Yavuz DG, Ozben B, Telli A, Ogunc AV, Yuksel M, Toprak A, Yazici D, Sancak S, Deyneli O et al. 2009 Effect of vitamin D deficiency and replacement on endothelial function in asymptomatic subjects. Journal of Clinical Endocrinology and Metabolism 94 4023-4030. (doi:10.1210/jc.2008-1212)

Thompson DD, Simmons HA, Pirie CM \& Ke HZ 1995 FDA guidelines and animal models for osteoporosis. Bone 17 125S-133S. (doi:10.1016/ 8756-3282(95)97353-H)

Tiyerili V, Muller CF, Fung S, Panek D, Nickenig G \& Becher UM 2012 Estrogen improves vascular function via peroxisome-proliferatoractivated-receptor- $\gamma$. Journal of Molecular and Cellular Cardiology $\mathbf{5 3}$ 268-276. (doi:10.1016/j.yjmcc.2012.05.008)

Widlansky ME, Gokce N, Keaney JF Jr \& Vita JA 2003 The clinical implications of endothelial dysfunction. Journal of the American College of Cardiology 42 1149-1160. (doi:10.1016/S0735-1097(03)00994-X)

Yeboah J, Crouse JR, Hsu FC, Burke GL \& Herrington DM 2007 Brachial flow-mediated dilation predicts incident cardiovascular events in older adults: the Cardiovascular Health Study. Circulation 115 2390-2397. (doi:10.1161/CIRCULATIONAHA.106.678276)

Yiu YF, Chan YH, Yiu KH, Siu CW, Li SW, Wong LY, Lee SW, Tam S, Wong EW, Cheung BM et al. 2011 Vitamin D deficiency is associated with depletion of circulating endothelial progenitor cells and endothelial dysfunction in patients with type 2 diabetes. Journal of Clinical Endocrinology and Metabolism 96 E830-E835. (doi:10.1210/ jc.2010-2212)

Received in final form 29 October 2015

Accepted 3 November 2015

Accepted Preprint published online 4 November 2015 http://joe.endocrinology-journals.org DOI: 10.1530/JOE-15-0332
(C) 2016 Society for Endocrinology Printed in Great Britain
Published by Bioscientifica Ltd. 